\title{
Detection and analysis of coherent structures in urban turbulence
}

\author{
C. Feigenwinter and R. Vogt
}

With 6 Figures

Received October 27, 2003; revised October 15, 2004; accepted November 16, 2004

Published online February 16, 2005 (C) Springer-Verlag 2005

\begin{abstract}
Summary
The continuous wavelet transform provides a suitable tool to visualize the vertical structure of turbulence and to detect coherent structures in turbulent time series. This is demonstrated with a simple example of an artificially ramp structured time series. In this study turbulence data, i.e. the fluctuations of the horizontal wind components $u^{\prime}$ and $v^{\prime}$, the vertical component $w^{\prime}$ and temperature $T^{\prime}$, sampled with $20.83 \mathrm{~Hz}$ and measured simultaneously at three levels $(z / h=1.5,2.1$ and 3.2, with $z$ as the sensor height and $h$ the height of the roughness elements) over an urban canopy in the inner city of Basel, Switzerland, are analyzed. The detection of the coherent structures was performed using the Mexican hat wavelet and the zero-crossing method. The analysis for unstable conditions shows that organized structures (ejection-sweep cycles) cover about $45 \%$ of the total run time. A conditional average from a total of 116 detected ejection-sweep sequences during 7 hours was calculated over a time window of 100 seconds. This dominating time scale was derived from peak frequencies of the wavelet spectra as well as from the Fourier spectra. It is shown that the normalized amplitudes of fluctuations of temperature and longitudinal wind speed during the events are largest at the lowest measurement level just above the canopy and decrease with increasing distance from the roughness elements. A comparison of related studies over different non-urban surfaces (mainly forests) shows that the shape of conditionally averaged ejectionsweep sequences is very similar for all canopies, however, the dominating time scale in general increases the rougher the surface is and the higher the roughness elements are.
\end{abstract}

\section{Introduction}

One of the main goals of studying urban turbulence is to understand the processes that rule the exchange of momentum, heat and mass between the urban surface and the atmosphere and its effect on the energy balance. Results of such studies e.g. help to improve the modeling of pollutant dispersion which is one of the most important applications in urban meteorology. Because of the rough and inhomogeneous urban surface problems arise when applying traditional micrometeorological concepts such as profile methods (e.g. Oke, 1987) or the MoninObukhov similarity (MOS) theory (Monin and Obukhov, 1954). Nevertheless, because of the lack of a unifying method for turbulent transfer, recent investigations of urban turbulence have focused on testing the above mentioned methods in urban boundary layer experiments and describing their specific departures from studies made over ideal surfaces in terms of homogeneity, fetch conditions and roughness. In his comprehensive review article Roth (2000) gives an overview on the recent work of turbulence observations over cities by analyzing more than fifty studies dealing with the subject. He concludes that results on integral statistics and spectra show a strong correlation between turbulent 
flows over cities and plant canopies and that this results may be interpreted in the framework of plane mixing-layer flows (Raupach et al., 1996) rather than in MOS or by gradient methods. He also emphasizes that future urban turbulence research should, beside other topics, focus on organized (coherent) motions which manifest in ejection-sweep cycles and ramp structures in turbulent time series. This is the point where the present study will contribute to a better understanding of turbulent transport processes over urban surfaces.

An increasing number of papers dealing with atmospheric turbulence and wavelets have been published since 1991, when Meneveau (1991) and Fargé (1992) established the wavelet transform in turbulence research. The continuous wavelet transform is an attractive tool for decomposing a time series into time-frequency space, which gives the opportunity to determine both the dominant modes of variability and how these modes vary in time. Turbulent transport within and above a vegetation canopy is dominated by large-scale intermittent coherent structures. These structures are known as periodic ramp patterns in time series of scalars (e.g. temperature) or, in a more general point of view, as occasional large amplitude excursions from the mean in time series of turbulent variables. They are characterized by cycles of 'ejections' (bursts) and 'sweeps' (gusts), which have been observed over a wide range of canopies.

Collineau and Brunet (1993a, b) proposed a suitable tool to detect ramp structures in turbulent time series based on the wavelet transform. In this paper we apply this method to detect and analyze organized motions in turbulence data measured above an urban surface. In the first section a theoretical introduction is given and applied to a simple artificial time series to demonstrate the effectiveness of the described method. It follows a short literature overview on the different concepts to detect ramp structures in turbulent time series and to determine the contribution of organized motions to the total flux. We then discuss the results of the averaged conditional samplings of first and second order moments and compare the result to findings of related studies.

\section{Methods}

\subsection{The continuous wavelet transform}

The continuous wavelet transform $W(s, \tau)$ of a real square integrable signal $f(t)$ with respect to an analyzing wavelet $\psi(t)$ can be defined as

$$
\begin{aligned}
W(s, \tau) & =\frac{1}{s} \int_{-\infty}^{+\infty} f(t) \psi\left(\frac{t-\tau}{s}\right) d t \\
& =\frac{1}{s} \int_{-\infty}^{+\infty} f(t) \psi_{s, \tau}(t) d t,
\end{aligned}
$$

where $s$ is a scale dilation and $\tau$ is a position translation. It can be viewed as a numerical microscope, whose optics, magnification and position are given by $\psi(t), s$ and $\tau$, respectively (Holschneider, 1995).

The wavelet transform is an energy preserving transformation, and so it follows, that the total energy $E_{f}$ of a function $f(t)$ can be written as

$$
\begin{aligned}
E_{f} & =\int_{-\infty}^{+\infty}|f(t)|^{2} d t=\int_{-\infty}^{+\infty}|\hat{f}(\omega)|^{2} d \omega \\
& =\frac{1}{C_{\psi}} \int_{0}^{+\infty} \frac{d s}{s} \int_{-\infty}^{+\infty}|W(s, \tau)|^{2} d \tau,
\end{aligned}
$$

where $C_{\psi}$ is a constant for each wavelet function and ' $\wedge$ ' denotes the Fourier transform. Equation (2) correlates the Fourier energy spectrum $E_{f}(\omega)$ to the wavelet spectrum $E_{f}(s)$. See Perrier et al. (1995) for a comprehensive theoretical discussion of the relation between Fourier and wavelet spectra. For a complete theory of the wavelet transform refer to e.g. Holschneider (1995) and for a more practical guide to wavelet analysis to Torrence and Compo (1998).

\subsection{Detection method - a simple example}

The analyzing wavelet in this study is the so called Mexican hat wavelet, a second derivative of a Gaussian. This wavelet has the advantage, that the detection of temperature ramps involves only the identification of zero-crossing points of the wavelet coefficients with a particular slope sign and does not require the use of a threshold like other methods. For a comprehensive test of temperature ramp detection methods see Collineau and Brunet (1993a). Additionally, the wavelet transform as an alternative to the Fourier spectral analysis can also be used to determine the characteristic time scales of the organized 

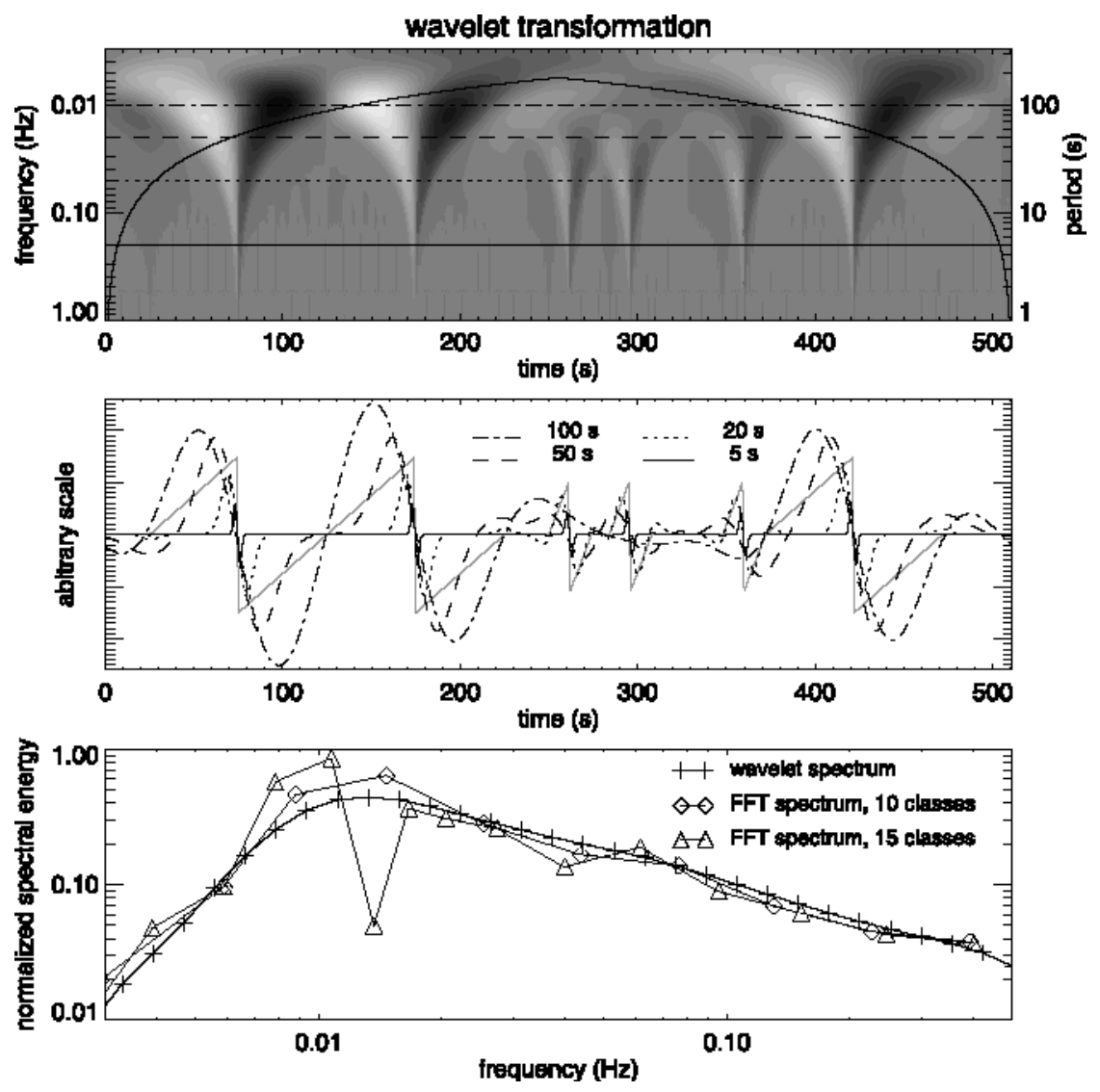

Fig. 1. Wavelet scalogram (top) and wavelet coefficients for scales corresponding to 5 (solid), 20 (dotted), 50 (dashed) and 100 (dashed-dotted) seconds of an artificial ramp data set (light gray) (center) and corresponding wavelet spectrum and Fourier spectra (bottom)

structures as shown by Collineau and Brunet (1993b). In the following a simple example demonstrates the capabilities of the continuous wavelet transform to detect organized structures and their characteristic time scale.

The wavelet transform has been applied on a signal of idealized ramps shown in Fig. 1 (gray line at center plot). The wavelet coefficients at different scales (or periods) show a zero-crossing at times, when a sharp decrease (jump) occurs, if the scale $s$ does not override the characteristic duration of the ramp (about 100s), which is determined by analyzing the wavelet variance (or spectrum) according to Eq. (2). The wavelet spectrum as well as the Fourier spectrum in Fig. 1 (bottom) exhibit a peak at the frequency that contributes most to the energy of the input signal, around $0.01 \mathrm{~Hz}$ in our example, which corresponds to a time-scale of 100 seconds. This plot is also a good example for the advantages of the (smoother) wavelet spectra compared to Fourier spectra that behave inconsistent in the low frequency range depending on the number of classes for averaging. Also the local properties of the wavelet transform cause the wavelet spec- trum to be dominated by local events rather than being the result of the entire signal, i.e. the peak of the wavelet spectrum represents the dominating width or duration of the events while the Fourier spectra peaks at the scale of the main periodicity, which includes the spacing between events (Mahrt and Howell, 1994).

It is therefore possible to physically interpret the wavelet variance peak as the dominant scale of coherent structures (e.g. Collineau and Brunet, 1993b; Lu and Fitzjarrald, 1994).

When analyzing turbulent time series it rarely happens that some events are missed or false detections are made. In any case, however, they do not affect the result, say the conditional averages, significantly, since the main structures are obviously catched by the detection algorithm.

The top of Fig. 1 represents the wavelet transform according to Eq. (1). The wavelet coefficients (contours) are plotted against time (ordinate) and frequency or scale (abscissa). Light colors represent positive, dark colors negative wavelet coefficients. This gives an idea of how to interpret such a plot with respect to the original input signal. Every sharp decrease is 
represented by a clear transition from light to dark. Low-amplitude ramps are represented by low amplitudes of the wavelet coefficients, resulting in more diffuse colors in the contour plot. The solid black line represents the "cone of influence". The region above this line is subject to errors due to edge effects of the Fourier transformation when applied to finite and non cyclic time series (Torrence and Compo, 1998).

Once we have detected the significant events by the zero crossing method, conditional averages sampled over a suitable time window $\Delta t$ (which corresponds to the dominating time scale) centered at detection points $t_{i}$ are calculated after

$$
\left\langle f^{\prime}(t)\right\rangle=\frac{1}{N} \sum_{N} \frac{f\left(t+t_{i}\right)-\overline{f_{i}}}{\sigma f_{i}}
$$

with $t_{i}-\Delta t / 2<t<t_{i}+\Delta t / 2, N$ the number of detection points, $\sigma f_{i}$ the standard deviation over the time window for normalization and $\bar{f}_{i}$ the local mean value. Similarly the conditionally averaged flux of a variable $f$ is calculated by

$$
\left\langle w^{\prime} f^{\prime}\right\rangle=\frac{1}{N} \sum_{N}\left(w\left(t+t_{i}\right)-\bar{w}_{i}\right)\left(f\left(t+t_{i}\right)-\bar{f}_{i}\right),
$$

and the ratio of the coherent structures to the total turbulent flux is consequently

$F_{\mathrm{coh}}=\frac{\left\langle w^{\prime} f^{\prime}\right\rangle}{\overline{w^{\prime} f^{\prime}}}$,

where $\overline{w^{\prime} f^{\prime}}$ is the total Reynolds averaged turbulent flux of variable $f$ (i.e. $\overline{w^{\prime} T^{\prime}}$ for kinematic sensible heat flux and $\overline{w^{\prime} u^{\prime}}$ for momentum flux).

\subsection{Other concepts}

A short overview of related studies is given in Table 2. There is a wide range of different methodologies and in the following we briefly discuss some results concerning the contributions of coherent structures to the total Reynolds flux.

Hagelberg and Gamage (1994) applied a decomposing of the total buoyancy flux into a flux due to velocity structures and a flux due to temperature structures. For a $210 \mathrm{~s}$ aircraft data set from $600 \mathrm{~m}$ a.g.l. in a convective boundary layer they obtain contributions of $64 \%$ and $74 \%$, respectively, using a decomposition technique based on a wavelet transform that preserves coherent structures. Collineau and Brunet (1993b) and Bergström and Högström (1989) applied a triple decomposition of the turbulent variables into a long-term average, a perturbation due to large-scale motion and the remaining small-scale fluctuation. Collineau and Brunet (1993b), using the zero-crossing detection method, found contributions of $26 \%$ and $40 \%$ for momentum and sensible heat flux, respectively. Bergström and Högström (1989) determined the part of the total fluxes accounted for by the coherent structures as $92.7 \%$ and $87.5 \%$ for momentum and sensible heat flux, respectively, as an average for the three heights. Note that they took into account the varying lengths of the detected events, and the conventionally derived fluxes were only calculated for the ramp periods. Howell and Mahrt (1994) proposed a partitioning into four modes (mesoscale, large eddy scale, transporting eddy scale and fine scale) using the Haar and the D4 wavelet. Largest contributions (about 50\%) to the total momentum flux from a 9.1 hours data set obtained $45 \mathrm{~m}$ above flat terrain were found in the transporting eddy mode, defined by a length scale ranging from $100 \mathrm{~m}$ to $600 \mathrm{~m}$. Lu and Fitzjarrald (1994) used the Haar function as wavelet for ramp detection in and above a deciduous midlatitude forest and found the coherent structures to contribute about $40 \%$ of the momentum and heat transport above the forest in their 85 hours data set, with equal importance for the upward and downward components. Qiu et al. (1995) used the Haar wavelet for ramp detection and a two step pseudo-wavelet function for the determination of the duration of coherent structures and for conditional sampling. They found that coherent structures contribute "heavily" to observed fluxes, but they also stated that subjectivity in choosing threshold durations causes differences in quantitative estimates of the structure contributions. A recent publication by Krusche and Oliveira (2004) found the intermittency function to best identify temperature ramps, followed by the Mexican hat wavelet transform in a 43 hours data set. A remarkable conclusion of their work is that, after applying a statistical test, the results of the two methods do not belong to the same population. Similar results are published by Yuan and Mokhtarzadeh-Dehghan (1994) who tested various conditional-sampling techniques and found on the one hand good correspondence 
between events detected by some methods but on the other hand large discrepancies suggesting that not all techniques detect the same process.

The overview is far from being complete, but it demonstrates the wide variety of databases and methods for the detection of organized motions and for the determination of their contribution to the total flux. This has to be taken into account when comparing the results of different studies.

\section{Results and discussion}

Data analyzed in this study are from a special measurement campaign, which took place in the inner city of Basel, Switzerland, from July 25, 1995, to February 27, 1996. Three Solent Gill ultrasonic anemometers (Type R2, sampling rate $20.83 \mathrm{~Hz}$ ) were operated at three different heights $(z / h=3.2$,
2.1 and 1.5 ) on a $51 \mathrm{~m}$ antenna tower on the roof of a $24 \mathrm{~m}$ high office building. See Feigenwinter et al. (1999) and Feigenwinter (2000) for a detailed description of the experimental setup.

A cluster of 7 runs (DOY 215, 12:00 to 19:00) under unstable conditions was chosen for the analysis of the Fourier and wavelet temperature spectra. Meteorological conditions were very similar for all hours (see Table 1), with steady moderate winds blowing from North-NorthWest, temperatures around $27^{\circ} \mathrm{C}$ and unstable conditions. The analysis shows that the peak frequencies of the temperature spectra lie in the range from 0.009 to $0.015 \mathrm{~Hz}$, with a slight shift to lower frequencies with increasing height as shown in Fig. 2. Therefore, the scale chosen for jump detection corresponds to a period of about 90 seconds. Jump detection was performed at

Table 1. Statistics of event detection and meteorological conditions for DOY 215 from 12:00 to 19:00, with $\Delta t_{e v}$ as the mean duration between events, $N_{e v}$ the number of detected events, $T$ the mean air temperature, $R H$ the relative humidity, $U$ the mean horizontal wind velocity, $W D$ the mean wind direction, $u^{*}$ the friction velocity, $H$ the sensible heat flux and $\left(z-z_{d}\right) / L$ the stability index

\begin{tabular}{llllllllll}
\hline Time & $\Delta t_{e v}$ & $N_{e v}$ & $\begin{array}{l}T_{z / h=1.5} \\
{ }^{\circ} \mathrm{C}\end{array}$ & $\begin{array}{l}R H_{z / h=1.5} \\
\%\end{array}$ & $\begin{array}{l}U_{z / h=3.2} \\
\mathrm{~m} \mathrm{~s}^{-1}\end{array}$ & $\begin{array}{l}W D_{z / h=3.2} \\
\circ\end{array}$ & $\begin{array}{l}u_{z / h=3.2}^{*} \\
\mathrm{~m} \mathrm{~s}^{-1}\end{array}$ & $\begin{array}{l}H_{z / h=3.2} \\
\mathrm{~W} \mathrm{~m} \mathrm{~m}^{-2}\end{array}$ & $\begin{array}{l}\left(z-z_{d}\right) / L \\
\text { at } z / h=3.2\end{array}$ \\
\hline $13: 00$ & $196 \pm 102 \mathrm{~s}$ & 16 & 25.6 & 45 & 3.45 & 330 & 0.31 & 255 & -4.88 \\
$14: 00$ & $193 \pm 58 \mathrm{~s}$ & 15 & 26.7 & 42 & 3.30 & 326 & 0.26 & 303 & -10.56 \\
$15: 00$ & $178 \pm 108 \mathrm{~s}$ & 17 & 27.5 & 40 & 3.49 & 346 & 0.40 & 322 & -2.98 \\
$16: 00$ & $192 \pm 129 \mathrm{~s}$ & 16 & 27.7 & 40 & 3.33 & 344 & 0.42 & 319 & -2.47 \\
$17: 00$ & $227 \pm 149 \mathrm{~s}$ & 13 & 27.7 & 40 & 5.60 & 338 & 0.51 & 200 & -0.90 \\
$18: 00$ & $170 \pm 65 \mathrm{~s}$ & 19 & 27.1 & 43 & 6.20 & 342 & 0.70 & 207 & -0.35 \\
$19: 00$ & $149 \pm 71 \mathrm{~s}$ & 20 & 26.1 & 46 & 5.95 & 336 & 0.59 & 80 & -0.22 \\
\hline
\end{tabular}

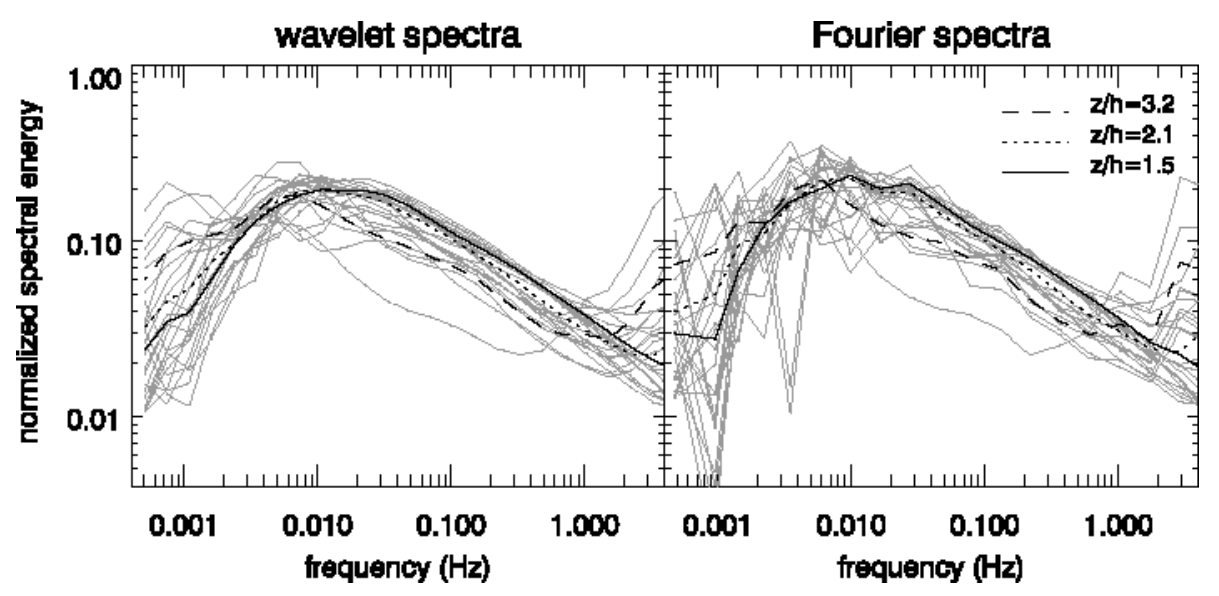

Fig. 2. Comparison of averaged (black lines, solid for $z / h=1.5$, dotted for $z / h=2.1$ and dashed for $z / h=3.2$ ) wavelet (left) and Fourier (right) spectra for DOY 215 from 12:00 until 19:00 for temperature fluctuations to determine the dominant time scale for event detection. Dominant time scale was taken from the peak of the wavelet temperature spectrum at $z / h=1.5$. Light gray lines correspond to spectra derived from single runs at all heights 
$z / h=1.5$. Detection points had to occur simultaneously within an interval of $\pm 25 \mathrm{~s}$ at the upper two levels for the computation of the conditional averages after Eq. (3).

Ramp structures can clearly be detected by eye in the temperature time series in Fig. 3, where periods of slow increase are followed by an abrupt decrease. These ramps occur simultaneously at all three levels but also isolated at just one or two levels. For this study, we concentrate on simultaneous events at all three levels, because we are interested in the main structures that dominate the exchange. Figure 3 shows also the scalogram of the continuous wavelet transform of an arbitrarily chosen 53 minutes run ( $2^{16}$ data points) of temperature fluctuations $T^{\prime}$ at three levels. Zero crossing points of the wavelet transform coefficients with a negative slope for different periods ranging from 30 to 400 seconds are indicated by small white points (black for the $90 \mathrm{~s}$ period). The simultaneous events detected at $z / h=1.5$ that contribute to the conditional averages for the $90 \mathrm{~s}$ period, are marked by large white

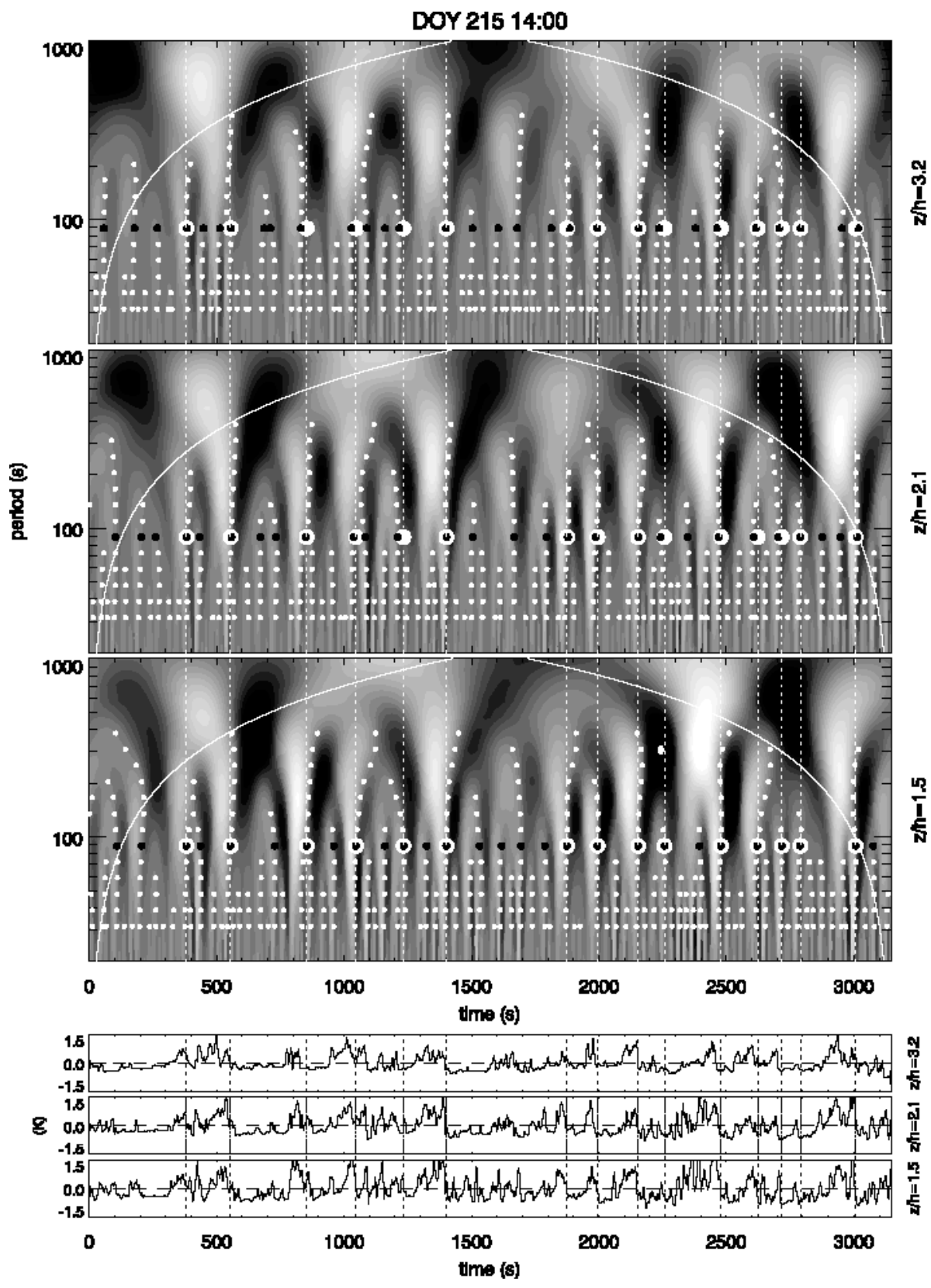

Fig. 3. Wavelet transform of temperature fluctuations $T^{\prime}$ at three different heights $(z / h=3.2$, 2.1 and 1.5, upper part from top to bottom) and the corresponding time series (lower part) with detected simultaneous events. Small white points mark the zero crossings of the wavelet coefficients for periods from 30 to 400 seconds. Black circles mark all events detected at the dominant time scale of $90 \mathrm{~s}$ and the large white circles mark the simultaneous events taken for the conditional averages 


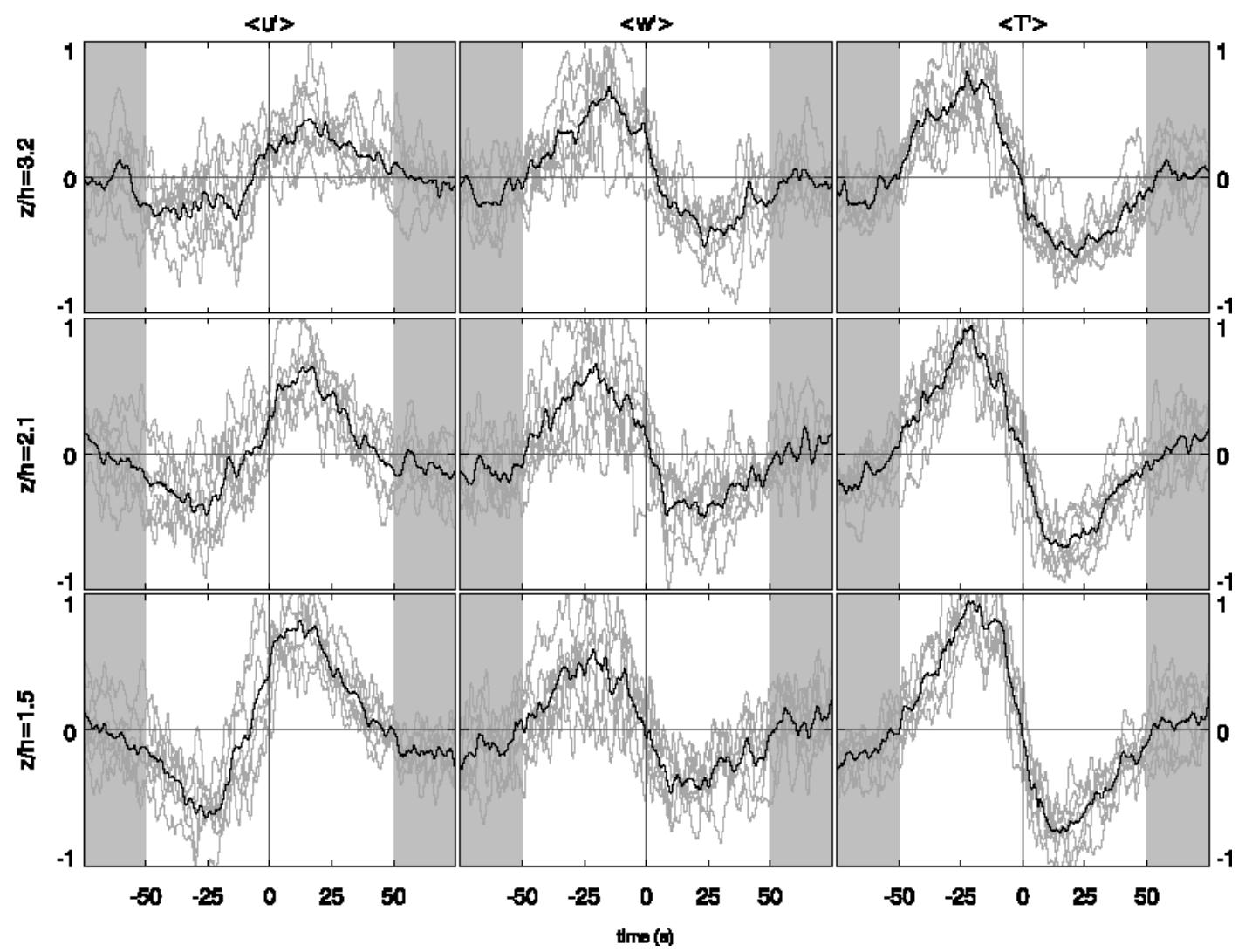

Fig. 4. Conditional averages of $\left\langle u^{\prime}\right\rangle,\left\langle w^{\prime}\right\rangle$ and $\left\langle T^{\prime}\right\rangle$ (from left to right) and heights $z / h=3.2,2.1$ and 1.5 (from top to bottom) for DOY 215 from 12:00 to 19:00. Light gray lines are the results for every single run. Data are normalized with their respective standard deviations according to Eq. (3) and smoothed with a $1 \mathrm{~s}$ running mean. Shaded regions do not contribute to the conditionally averaged second order moments $\left\langle u^{\prime} w^{\prime}\right\rangle$ and $\left\langle w^{\prime} T^{\prime}\right\rangle$

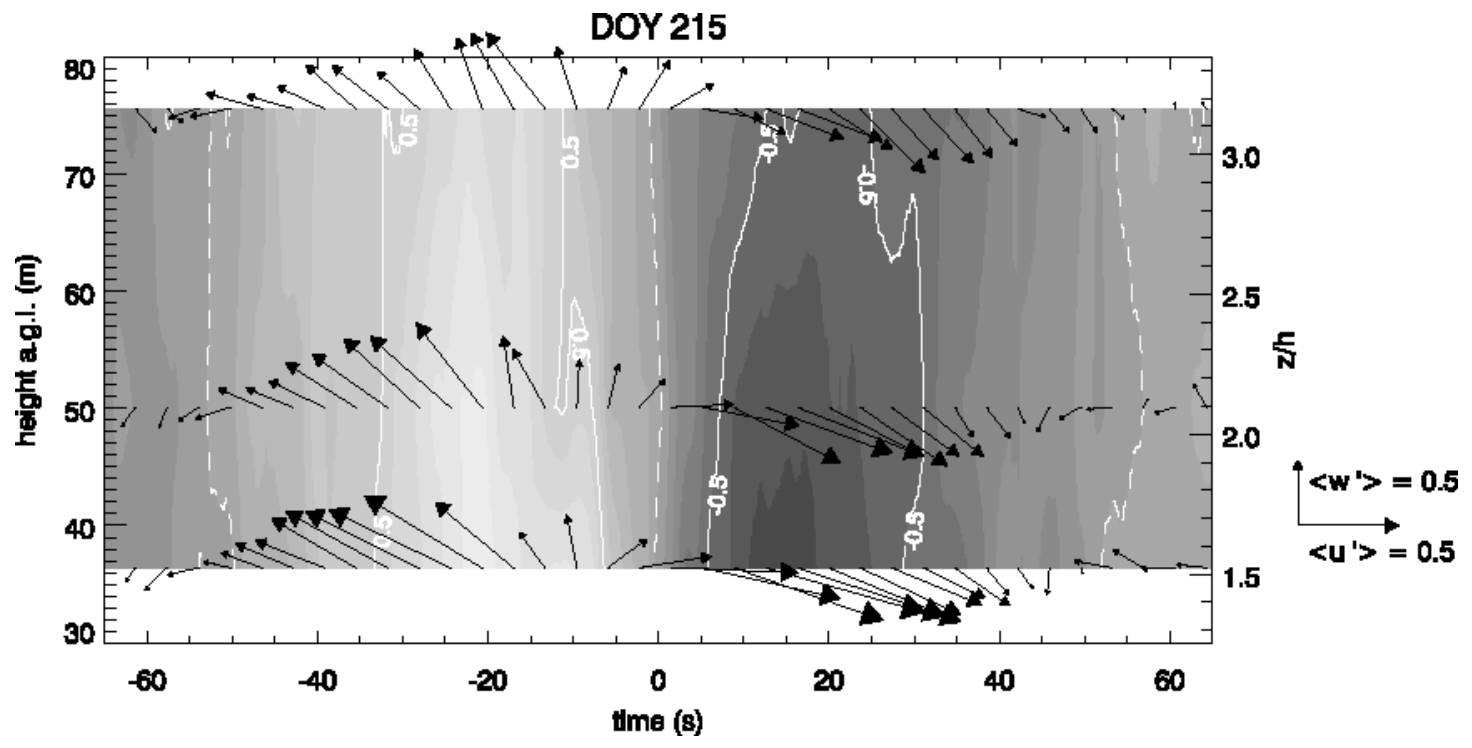

Fig. 5. Vertical cross section of the ensemble normalized temperature (contours) and wind (arrows) field of the organized structures derived from 116 events between 10:00 to 21:00 on DOY 215 under unstable conditions. Data are normalized with their respective standard deviations according to Eq. (3) and smoothed with a $1 \mathrm{~s}$ running mean 
circles in the contour plot and as dashed lines in the time series. This figure shows several important details when applying the detection method to "real world" data. The number of detected events for conditional sampling is of course heavily dependent on the chosen time scale and, more relevant, on the definition of "simultaneousness" ( $\pm 25 \mathrm{~s}$ in our case $)$. This will have further impact on the determination of the contribution of the detected events to the total flux. Also, the detected events are of different shape and duration which reflects the chaotic nature of turbulence.

The mentioned procedure results in a total of 116 detected events between 12:00 and 19:00, an average of 16 events per 53 minute run. Thus organized motions cover about $45 \%$ of the total period. An important feature derived from detection statistics printed in Table 1 is the mean time-interval between events $\Delta t_{e v}$ and, of course, the number of detected events per run $N_{e v}$. Though the mean duration between events is in a reasonable range, the large standard deviations suggest, that there are series of consistent events (of period shorter than $\Delta t_{e v}$ ) followed by longer "quiescent" periods. Thus $\Delta t_{e v}$ only gives a crude idea of the distribution of temperature ramps during a single run and is of course dependent on the number of detected events $N_{e v}$, which itself shows a moderate runto-run variation.

\subsection{First order moments}

For the 116 detected events, the conditional sampling procedure according to Eq. (3) results in an average pattern of a typical ejection-sweep sequence for unstable conditions shown in Figs. 4

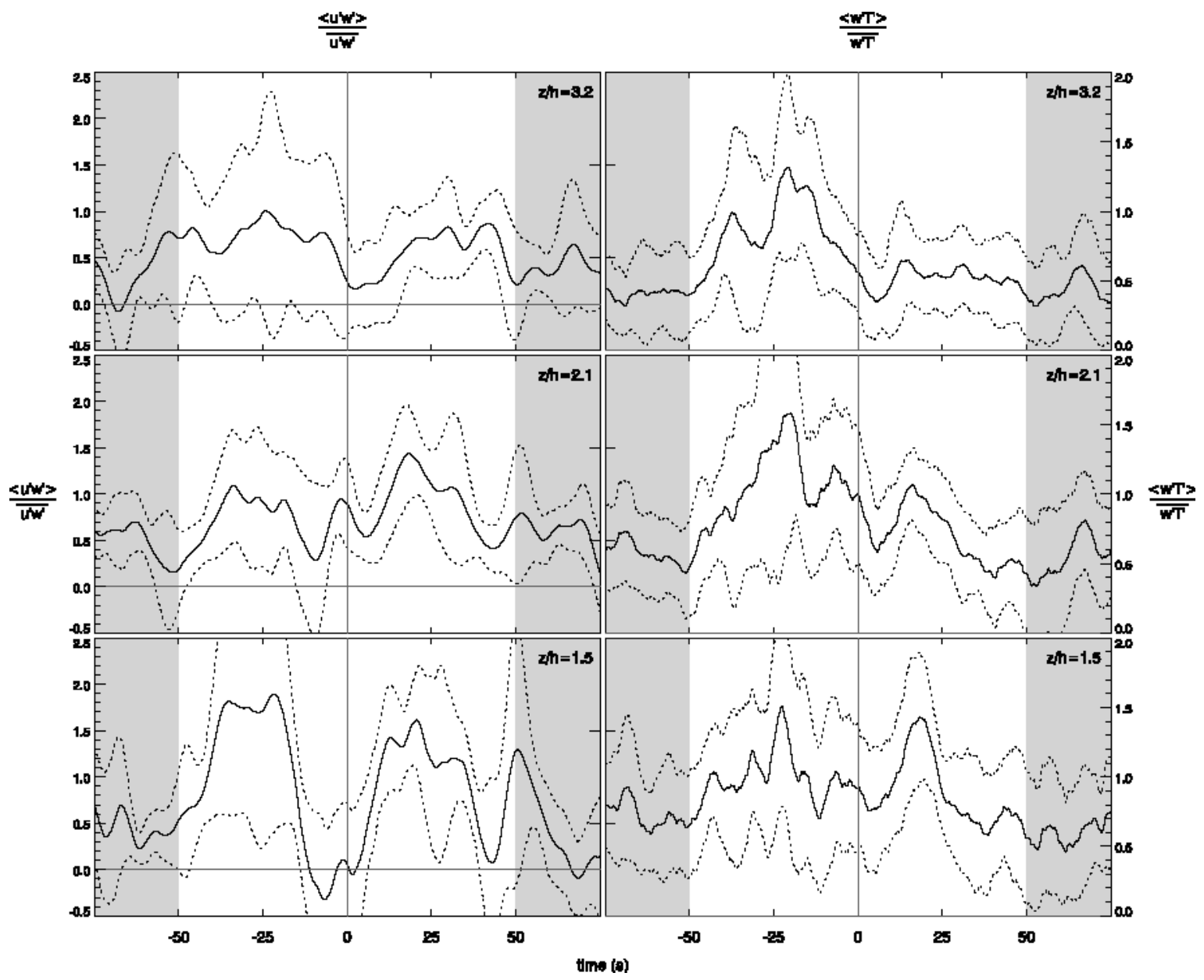

Fig. 6. As Fig. 4 but for ratios of conditionally averaged second-order moments to the total flux. From top to bottom: $z / h=3.2,2.1$ and 1.5. Left: $\left\langle u^{\prime} w^{\prime}\right\rangle / \overline{u^{\prime} w^{\prime}}$. Right: $\left\langle w^{\prime} T^{\prime}\right\rangle / \overline{w^{\prime} T^{\prime}}$. Data are smoothed with a $1 \mathrm{~s}$ running mean 
and 5. A time window of 130 seconds is chosen for the presentation to show also the evolution immediately before and after the event. Figure 4 shows all components each plotted in a single window whereas Fig. 5 shows the result in a more compact manner as a combination of a contour plot for temperature fluctuations and with arrows for the wind velocity. Both figures demonstrate how temperature and vertical and horizontal wind components interact during an ejection-sweep event. Temperature rises moderately towards the detection point, where a sharp decrease is followed by a steadily increase. Amplitudes of the fluctuations are largest at the lowest level. At relatively slow longitudinal wind speed $\left\langle u^{\prime}\right\rangle$, warm air from the surface is transported to higher levels (ejection, burst) by a positive vertical wind component $\left\langle w^{\prime}\right\rangle$. When flow turns from upward to downward, the horizontal wind velocity $\left\langle u^{\prime}\right\rangle$ increases and advected colder air is transported downward (sweep, gust). The mean duration of these events was found to be around 90 seconds, which is in good correspondence to Oikawa and Meng (1995) for suburban surface data. Similar patterns were obtained for other surface types, however with different dominating time scales, see Table 2 for a comparison. The values from these studies support the idea, that the height of the roughness elements is linked to the length of the dominating time scale in a sense that higher roughness elements cause larger structures. The time scale also increases with increasing $z / h$ as shown by the temperature spectra in Fig. 2 .

\subsection{Second order moments}

It is now of special interest to quantify the ratio of the detected structures to the total (Reynolds averaged) flux. Figure 6 shows the average ratio for momentum and kinematic heat flux for the 7 runs according to Eq. (5) and in Table 3 the results for every single run are listed. The detected structures are very efficient in transporting momentum and sensible heat, for some runs the ratio is even more than $100 \%$. This may happen when outward or inward interactions $\left(u^{\prime}\right.$ and $w^{\prime}$ positively correlated for momentum flux, $w^{\prime}$ and $T^{\prime}$ negatively correlated for sensible heat flux) account for a large part of the total flux. These interactions are not part of organized structures but directly affect the total flux. It is
Table 3. Ratios of conditionally sampled second order moments to total momentum flux and kinematic heat flux under unstable conditions

\begin{tabular}{llll}
\hline Time & $z / h$ & $\left\langle u^{\prime} w^{\prime}\right\rangle \overline{u^{\prime} w^{\prime}}$ & $\left\langle w^{\prime} T^{\prime}\right\rangle \overline{w^{\prime} T^{\prime}}$ \\
\hline $13: 00$ & 3.2 & 0.30 & 0.78 \\
& 2.1 & 0.42 & 1.05 \\
& 1.5 & 0.50 & 1.09 \\
$14: 00$ & 3.2 & 0.56 & 0.80 \\
& 2.1 & 0.82 & 1.24 \\
& 1.5 & 0.79 & 1.21 \\
$15: 00$ & 3.2 & 0.68 & 0.49 \\
& 2.1 & 1.07 & 0.76 \\
& 1.5 & 1.04 & 0.79 \\
$16: 00$ & 3.2 & 0.74 & 0.79 \\
& 2.1 & 0.62 & 0.92 \\
& 1.5 & 0.83 & 1.01 \\
$17: 00$ & 3.2 & 0.33 & 0.49 \\
& 2.1 & 0.40 & 0.62 \\
& 1.5 & 0.41 & 0.79 \\
$18: 00$ & 3.2 & 0.70 & 0.85 \\
& 2.1 & 0.73 & 0.84 \\
$19: 00$ & 1.5 & 0.82 & 0.89 \\
& 3.2 & 0.71 & 0.75 \\
& 2.1 & 0.76 & 0.87 \\
& 1.5 & 0.83 & 0.64 \\
\hline
\end{tabular}

also possible that some events overlap each other and thus are partly counted twice. Altogether this may raise the question whether the applied method is appropriate to determine the contribution of coherent structures to the total flux.

In general, the most efficient transport for both, momentum and sensible heat flux, occurs $\pm 25 \mathrm{~s}$ within the detection point, and the ratio decreases with increasing height. Compared to the steady patterns of first order moments, the scaled conditional averages of second order moments $\left\langle u^{\prime} w^{\prime}\right\rangle / \overline{u^{\prime} w^{\prime}}$ and $\left\langle w^{\prime} T^{\prime}\right\rangle / \overline{w^{\prime} T^{\prime}}$ show a large scatter (shown as curves of standard deviations in Fig. 6) which originates from the variability in shape and length of the single events.

\section{Summary and conclusions}

The continuous wavelet transform provides a suitable tool to visualize the vertical structure of turbulence and to detect coherent structures in turbulent time series. This is demonstrated with a simple example of a ramp structured time series. The analysis of turbulence data from above an urban canopy under unstable conditions 
shows, that organized structures cover about $45 \%$ of the total run time. Conditional averages from a total of 116 detected ejection-sweep sequences were calculated over a time window of $100 \mathrm{~s}$. It is shown, that the normalized amplitudes of fluctuations of temperature and longitudinal wind speed during the events are largest just above the canopy and decrease with increasing distance from the roughness elements. A comparison of related studies over different canopies shows, that the shape of conditionally averaged ejectionsweep sequences is very similar for all canopies. The dominating time scale in general is increasing the rougher the surface is and the higher the roughness elements are.

Coherent structures are not only a feature of various types of vegetation canopies but can also be detected over rough urban surfaces by the same methods. For further experimental design it would be interesting to see how the structures propagate into the urban canopy, say street canyons. Despite the methodical difficulties to define the contribution of the coherent structures to the total flux it is obvious that they are very efficient in transporting momentum and sensible heat. The presented results are relevant for testing and verification of LES models. However, the large variety of detection methods, conditional sampling procedures and definitions for the partitioning of the total flux makes it difficult to compare the results of the relevant studies. Numerous concepts exist but none of them has been established as a standard until now. There is certainly a need for further work in this field to make the results of the different studies comparable.

\section{Acknowledgements}

The comments of the reviewers are highly appreciated. Funding for this study was provided by the Swiss National Science Foundation grants 20-40621.94 and 20-49490.96. Our thanks go to Balcab Kabelfernsehen Basel AG, Basel, for giving us the permission to use the antenna tower and their facilities for the data acquisition equipment.

\section{References}

Barthlott C (2003) Kohärente Wirbelstrukturen in der atmosphärischen Grenzschicht. Ph.D. thesis, Wiss Ber Inst Met Klim Nr. 33, Karlsruhe: University of Karlsruhe, 128 pp

Bergström H, Högström U (1989) Turbulent exchange above a pine forest, II: Organized structures. Bound-Layer Meteor 49: 231-263
Brunet Y, Collineau S (1994) Wavelet analysis of diurnal and nocturnal turbulence above a maize crop. In: FoufoulaGeorgiou E, Kumar P (eds) Wavelets in geophysics. San Diego: Academic Press, pp 129-150

Collineau S, Brunet Y (1993a) Detection of turbulent coherent motions in a forest canopy Part I: Wavelet analysis. Bound-Layer Meteor 65: 357-379

Collineau S, Brunet Y (1993b) Detection of turbulent coherent motions in a forest canopy Part II: Timescales and conditional averages. Bound-Layer Meteor 66: 49-73

Fargé M (1992) Wavelet transforms and their applications to turbulence. Annu Rev Fluid Mech 24: 395-457

Feigenwinter C (2000) The vertical structure of turbulence above an urban canopy. Ph.D. thesis, stratus 7, Basel: University of Basel, $76 \mathrm{pp}$

Feigenwinter C, Vogt R, Parlow E (1999) Vertical structure of selected turbulence characteristics above an urban canopy. Theor Appl Climatol 62: 51-63

Gao W, Shaw RH, Paw UKT (1992) Conditional analysis of temperature and humidity microfronts and ejection/ sweep motions within and above a deciduous forest. Bound-Layer Meteor 59: 35-57

Hagelberg CR, Gamage NKK (1994) Structure-preserving wavelet decompositions of intermittent turbulence. Bound-Layer Meteor 70: 217-246

Holschneider M (1995) Wavelets: an analysis tool. Oxford: Clarendon Press, 423 pp

Howell JF, Mahrt L (1994) An adaptive decomposition: Application to turbulence. In: Foufoula-Georgiou E, Kumar P (eds) Wavelets in geophysics. San Diego: Academic Press, pp 107-128

Krusche N, de Oliveira AP (2004) Characterization of coherent structure in the atmospheric surface layer. Bound-Layer Meteor 110: 191-211

Lu CH, Fitzjarrald DR (1994) Seasonal and diurnal variations of coherent structures over a deciduous forest. Bound-Layer Meteor 69: 43-69

Mahrt L, Howell JF (1994) The influence of coherent structures and microfronts on scaling laws using global and local transforms. J Fluid Mech 260: 247-270

Meneveau C (1991) Analysis of turbulence in the orthonormal wavelet representation. J Fluid Mech 232: 469-520

Monin AS, Obukhov AM (1954) Dimensionless characteristics of turbulence in the surface layer. Trudy Geofiz. Inst., Akad. Nauk SSR 24: 163-187

Oikawa S, Meng Y (1995) Turbulence characteristics and organized motions in a suburban roughness sublayer. Bound-Layer Meteor 74: 289-312

Oke TR (1987) Boundary layer climates. London, UK: Methuen

Paw UKT, Brunet Y, Collineau S, Shaw RH, Maitani T, Qiu J, Hipps L (1992) On coherent structures in turbulence above and within agricultural plant canopies. Agric Forest Meteorol 61: 55-68

Perrier V, Philipovitch T, Basdevant C (1995) Wavelet spectra compared to Fourier spectra. J Math Phys 36: 1506-1519 
Qiu J, Paw UKT, Shaw RH (1995) Pseudo-wavelet analysis of turbulence patterns in three vegetation layers. BoundLayer Meteor 72: 177-204

Raupach MR, Finnigan JJ, Brunet Y (1996) Coherent eddies and turbulence in vegetation canopies: The mixing-layer analogy. Bound-Layer Meteor 78: 351-382

Roth M (2000) Review of atmospheric turbulence over cities. Quart J Roy Meteor Soc 126: 941-990

Torrence C, Compo GP (1998) A practical guide to wavelet analysis. Bull Amer Meteor Soc 97(1): 61-78

Vogt R, Feigenwinter C (2000) Structure of turbulent exchange above a forest. In: de Dear RJ, Kalma JD, Oke TR, Aulicienms A (eds) Biometeorology and urban climatology at the turn of the millennium - Selected papers from the conference ICB-ICUC'99, Sidney, WMO/TD No. 1026, 211-216

Yuan YM, Mokhtarzadeh-Dehghan MRA (1994) Comparison study of conditional sampling methods used to detect coherent structures in turbulent boundary layers. Phys Fluids 6: 2038-2057

Authors' address: C. Feigenwinter (e-mail: christian. feigenwinter@unibas.ch), R. Vogt, Institute of Meteorology, Climatology and Remote Sensing, University of Basel, Klingelberstrasse 27, 4056 Basel, Switzerland. 